\title{
Las justificaciones de la muerte asistida
}

\section{The justifications for assisted death}

JOSÉ ANTONIO CERRILLO VIDAL (Universidad de Córdoba)

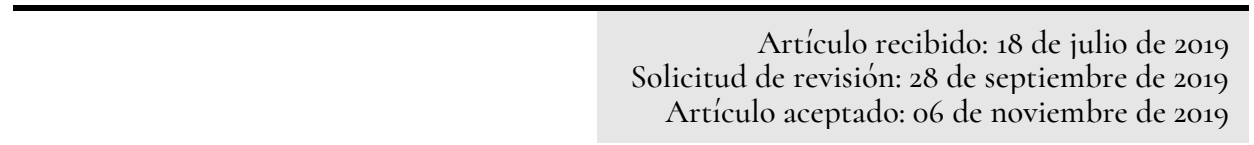

Cerrillo Vidal, José Antonio (2020). Las justificaciones de la muerte asistida. Recerca. Revista de Pensament i Análisi, 25(2), pp. 137-156.

\section{Resumen}

El concepto de muerte asistida comprende una heterogénea variedad de prácticas, cuyo denominador común es garantizar al ciudadano su derecho a participar en las decisiones relacionadas con su propio proceso de muerte, para que sea tan acorde a sus preferencias como sea posible. Se trata de uno de los temas más polémicos y discutidos en las últimas décadas, tanto en el ámbito académico como en la opinión pública. En este artículo se examinan las dos principales justificaciones favorables a su legalización: garantizar la autonomía individual como en los demás ámbitos de la vida y evitar un sufrimiento innecesario al moribundo. Se argumentará que, si bien ambas se encuentran relacionadas y en las dos se anudan la ética del cuidado con la de la autonomía del sujeto, la justificación por la autonomía es más sólida y resiste mejor las objeciones de los contrarios a la legalización de este derecho.

Palabras clave: proceso de muerte, eutanasia, suicidio asistido, justificaciones, autonomía individual, sufrimiento.

\begin{abstract}
The concept of assisted death includes a heterogeneous variety of practices, whose common denominator is to guarantee citizens their right to participate in decisions related to their own death process, to make it as consistent with their preferences as possible. It's one of the most controversial and discussed topics in recent decades, both in the academic field and in public opinion. This paper examines the two main justifications for its legalization: to guarantee individual autonomy as in other areas of life, and to avoid unnecessary suffering to the dying. It will be argued that, although both are related and both tie the ethics of care with the autonomy of the subject, the justification for autonomy is stronger and resists better the objections of those opposed to the legalization of this right.
\end{abstract}

Key Words: dying process, euthanasia, assisted suicide, justifications, individual autonomy, suffering. 


\section{INTRODUCCIÓN}

El debate sobre la muerte asistida es uno de los más polémicos y discutidos de las últimas décadas. Ha levantado fuertes controversias en la opinión pública y ha ocupado un lugar central en el derecho sanitario, la bioética y la filosofía moral contemporáneas. A fin de contribuir a una comprensión más racional de la problemática, este texto ${ }^{1}$ se propone repasar las principales líneas argumentales en defensa de la muerte asistida e identificar aquellas que justifiquen su legalización de manera más sólida. Para ello, se comenzará contextualizando el origen y la naturaleza del debate; a continuación, se definirán con precisión las distintas prácticas asociadas a la muerte asistida, profundizando en las implicaciones éticas y legales de cada una; más tarde se examinarán en detalle los principales argumentos favorables a la legalización de la muerte asistida, tratando los puntos fuertes y débiles de cada uno para, finalmente, discutir cuáles resultan más consistentes para su fundamentación como derecho ciudadano.

\section{LA MUERTE COMO PROCESO}

La muerte es más que el momento final de nuestra vida: morir es un proceso. Un proceso que comienza antes y se prolonga después del deceso propiamente dicho. Tras el fallecimiento del sujeto acontecen los rituales funerarios. $\mathrm{Su}$ función es cortar definitivamente el lazo con el fallecido, reintegrar a los supervivientes en la vida cotidiana y restaurar la normalidad del mundo de los vivos, la creencia en nuestra capacidad de control sobre la naturaleza y en el orden que atribuimos al universo, los cuales se han visto amenazados por la muerte. El tiempo que transcurre entre la muerte y la consumación del funeral es, por tanto, un periodo de transición, en el que el muerto aún no ha terminado de marchar. De ahí que en los funerales se juegue con la conexión entre la vida y la muerte, mezclando elementos de ambas: lo puro y lo impuro, lo normal y lo anormal, lo limpio y lo sucio, lo biológico y lo cultural, el cuerpo y la trascendencia del mismo (Douglas, 1973; Seale, 1998: 64-71). En las sociedades contemporáneas este proceso se ha reducido a su mínima expresión, pero en las culturas premodernas solía dilatarse incluso durante años, especialmen-

Una primera versión de este artículo fue presentada como comunicación en el VI Congreso de la Red Española de Políticas Sociales, celebrado en Sevilla, el 17 de febrero de 2017. 
te si se practicaban las dobles exequias, una costumbre muy extendida ${ }^{2}$ por la que se esperaba a que el cadáver se librase de sus impurezas corporales (la carne y los fluidos vitales) para proceder a su despedida definitiva en un segundo y con frecuencia más ostentoso funeral. Los toraja de la isla indonesia de Célebes, por ejemplo, conviven con el cadáver embalsamado, hasta el punto de servirle alimento como al resto de la familia, mientras reúnen los recursos necesarios para celebrar el funeral definitivo, lo cual puede tardar incluso años debido a los muchos gastos que comporta, incluyendo el sacrificio de numerosos bueyes y la organización de peleas de gallos (Grillo, 2008).

Igualmente, la muerte empieza antes de que acontezca la defunción. Comienza, de hecho, en el mismo momento en que el agente comienza a cuestionar su lugar en el mundo de los vivos. Así, un individuo puede considerar cercano su final por padecer una enfermedad terminal o, más generalmente, por la sensación de soledad y cansancio que conlleva la pérdida de la pareja y otros seres queridos de su misma generación. Lo que le hace sentir que su tiempo en el mundo ha terminado y que la muerte es preferible a seguir viviendo (Dworkin, 1994: 3; Kellehear, 2009: 14-16). Entonces, el moribundo comienza a cortar los lazos que le unen al resto de seres humanos: se ásla, deja de participar en los rituales de la vida cotidiana y de tener en consideración las convenciones de comportamiento, descuida su higiene, apariencia y alimentación... Todo ello constituye una auténtica caída en la naturaleza desde la cultura, una muerte social que, en no pocas ocasiones, precede a la física (Seale, 1998: 149-171).

Ahora bien, ¿es legítimo intervenir en este proceso? O, dicho de otro modo, ¿es deseable acelerar la muerte de una persona, especialmente de aquellos que no desean seguir viviendo? Cada sociedad ha dado respuestas diferentes a esta pregunta. Entre los grupos humanos que sobreviven al límite de la subsistencia es habitual el abandono de los débiles, los ancianos o los enfermos. Al hacerlo buscan garantizar la continuidad del grupo, frecuentemente con la aquiescencia de los damnificados. Es un comportamiento documentado en numerosos grupos de cazadores-recolectores que habitan entornos especialmente hostiles, como los inuit, los sirionó de la Amazonia boliviana, los ojibwa del actual Canadá o los hotentotes y los san del Kalahari (Thomas, 1983: 430431). Pero también en contextos más contemporáneos, como el de las habitantes de las favelas del nordeste brasileño estudiadas por Nancy Scheper-Hughes

Disponemos de evidencias de su presencia en culturas de todo el planeta, como mostró Robert Hertz en su clásico estudio (1990: 18-54). 
(200o), cuya extrema pobreza les obligaba a dejar morir a sus hijos más débiles. Colectividades más prósperas y estables consideraban un privilegio de los ancianos, sobre todo aquellos especialmente sabios o poderosos, escoger el momento de su propia muerte antes de dejar de resultar una carga para el resto del grupo. Un ejemplo muy llamativo es el famoso ritual de los Señores de la Lanza dinka, del actual Sudán del Sur, por el que un viejo guerrero se entierra vivo en presencia de toda la aldea. Este acto se considera una auténtica victoria de la comunidad frente a la propia muerte (Thomas, 1983: 441-443). De la misma forma, en la Grecia y Roma clásicas se entendía que la vida debía ser más que la mera reproducción biológica: si no era posible disfrutar de una existencia sustantiva, no merecía la pena seguir viviendo. Por esta razón, el suicidio, el aborto e incluso ciertas formas de infanticidio eran tolerados y no se consideraban inmorales (Dowbiggin, 2007: 7-11; Manning, 1998: 6-12; Mystakidou et al., 2005). Las grandes religiones monoteístas, en cambio, condenan cualquier forma de acelerar la muerte por medios humanos, pues la vida es un don divino y solo a Dios corresponde tomarla (Dowbiggin, 2007: 16-20; Lewis, 2000: 70-74; Manning, 1998: 17-18). La defensa a ultranza de la vida ha sido también heredada por la moderna medicina científica, con consecuencias no previstas como veremos a continuación.

En las sociedades contemporáneas los increíbles avances en la ciencia y la tecnología médicas han prolongado nuestras vidas hasta límites impensables siglos atrás, dilatando y complejizando la propia muerte. Históricamente se trataba de un proceso no muy duradero, al que además no llegaban muchas personas. En cambio, en el presente tiende a alargarse incluso contra la voluntad de quienes lo experimentan. Y dado que nuestra sociedad tiene en la autonomía individual uno de sus valores centrales, es común que los ancianos sientan una gran angustia ante la creciente incapacidad para valerse por sí mismos, así como una enorme vergüenza por la pérdida de control sobre sus cuerpos. De ahí que algunos prefieran acelerar el proceso de muerte (evitando cada vez más abandonar sus hogares, limitando todo lo posible las relaciones con otros seres humanos, relajando los cuidados que les permitirían alargar aún más sus vidas... En otras palabras, dejándose morir) a extender una vida que consideran poco satisfactoria o digna. Prefieren, por tanto, no prolongar su vida biológica más allá de su vida social efectiva. El problema es que muchos de ellos no pueden hacerlo. A menudo, tanto las instituciones como el propio entorno social les presionan para que desistan en su actitud y se sometan a los tratamientos que les mantendrán con vida. Su conducta es percibida como desviada, indeseable o incluso cobarde. En algunos casos se llega incluso a con- 
siderar que el anciano ya no es capaz de tomar decisiones por sí mismo y se le institucionaliza, es decir, se le interna en instituciones que someten su comportamiento a rutinas normativas en las que puede intervenir poco o nada. Una situación que genera auténtico pánico entre muchos de nuestros mayores (Kellehear, 2009; Lawton, 1998; Seale, 1995, 1996, 1998: 162-170, 2004).

Por otra parte, los avances en la medicina nos enfrentan a una serie de realidades nuevas, prácticamente inéditas en la historia humana:

Personas con daños cerebrales severos conectadas a respiradores, tubos de alimentación, etc.; niños que nacen sin cerebro o solo con el tronco cerebral (anencefalia); mujeres embarazadas en estado de muerte cerebral; estados vegetativos persistentes o crónicos; técnicas de reproducción asistida; diagnóstico prenatal; trasplante de órganos y xenotrasplantes (Ausín y Peña, 1998: 15-6).

Situaciones de enorme complejidad moral y para las que apenas disponemos de guías o experiencias previas por su novedad, para las que estamos poco preparados colectivamente $\mathrm{y}$, más aun, individualmente. Varias de ellas se encuentran en la frontera que separa la vida de la muerte, un límite que la tecnología médica ha vuelto más y más borroso en el último siglo y medio, tanto que incluso el criterio científico para diagnosticar la muerte ha tenido que cambiarse en las últimas décadas. De la tradicional consideración de la parada cardiorrespiratoria se ha pasado a un incómodo consenso actual en torno a la muerte cerebral, que tampoco se ha visto exento de problemas ${ }^{3}$ (Singer, 1997: 33-49). En suma, vivimos una contradicción entre un sistema sanitario hipertecnificado, preparado para combatir la enfermedad y la muerte misma hasta donde sea posible, y una sociedad que todavía no cuenta con las herramientas suficientes para gestionar los limbos biológicos, éticos y jurídicos que aquel produce. Una contradicción en la que muchas personas quedan atrapadas, viéndose obligadas a padecer una muerte prolongada, dolorosa o simplemente opuesta a sus preferencias (Ausín y Peña, 1998).

3 De hecho, el criterio actual para la definición de muerte es la muerte cortical, la pérdida de las funciones superiores del cerebro, ya que el de simple muerte cerebral tenía el inconveniente de no incluir los estados vegetativos persistentes y los bebés anencefálicos (Salazar, 2001; Singer, 1997: 36 y ss.). 


\section{LA REIVINDICACIÓN DE LA MUERTE ASISTIDA}

Este es el caldo de cultivo en el que surgen las reivindicaciones por la legalización de la muerte asistida o, por usar el término acuñado por sus defensores, el derecho a una muerte digna. Podemos entender la muerte digna como

[...] aquella en la que el enfermo, que es un individuo autónomo, puede elegir libremente cómo desea morir (dentro de las posibilidades que se le ofrecen), y los profesionales de la sanidad deben respetar esa dignidad salvaguardando la libertad del paciente (Aguiar, Serrano Del Rosal y Sesma, 2009: 2).

Así pues, con la idea de la muerte digna se aspira a que el individuo decida libremente sobre el proceso de su propia muerte, reclamando un papel activo del ciudadano frente a la compleja realidad a la que nos enfrenta un sistema sanitario altamente tecnificado. El ejercicio autónomo de este derecho, a su vez, requiere que se den una serie de condiciones que han de ser garantizadas por los poderes públicos: un marco legal que reconozca el derecho a la libre elección del proceso de muerte y que exima de responsabilidades penales a los profesionales sanitarios que participen en él; información completa, veraz y confidencial acerca del diagnóstico y los tratamientos que se ofrecen a la persona próxima a la muerte; unos profesionales sanitarios comprensivos, respetuosos con las decisiones de sus pacientes y dispuestos a ayudarles a ponerlas en práctica, etc.

El derecho a una muerte asistida supondría un desarrollo de derechos ya reconocidos por el ordenamiento jurídico de todo país democrático, como el derecho a la libertad, a la dignidad, a la intimidad personal, al libre desarrollo de la personalidad y a la libertad ideológica y religiosa (Méndez Baiges, 2002: 53). Alude, asimismo, a un amplio conjunto de actuaciones con distintas consecuencias éticas y jurídicas y diferentes grados de aceptación social. Es preciso, por consiguiente, distinguirlas analíticamente (Aguiar, Serrano Del Rosal y Sesma, 2009: 2-4; De Miguel Sánchez y López Romero, 2006: 208-210; SimónLorda y Barrio-Cantalejo, 2008: 448-45o; Simón-Lorda et al., 2008):

1) La eutanasia, cuyo significado etimológico literal es 'buena muerte'. Es el término más extendido socialmente, el que más discusión ha suscitado y, por consiguiente, el que más se presta a confusión. En rigor, la eutanasia incluye toda actuación (sea por acción $u$ omisión) que provoca la muerte rápida e indolora de un paciente que $a$ ) padece una enfermedad incurable que le produce un sufrimiento o malestar físico o psicológico que considera inaceptable y que 
no es posible mitigar por cualquier otro medio y que $b$ ) disfrutando de completa capacidad de decisión y estando plenamente informado de las consecuencias, lo solicita de forma expresa y reiterada. Como puede observarse, la eutanasia implica de forma directa al personal sanitario: son sus prácticas la causa inmediata de la muerte de la persona que, cabe insistir, lo ha pedido expresa y reiteradamente con anterioridad. De lo contrario estaríamos ante un homicidio. Hay que destacar también que, en la actualidad, se considera irrelevante la tradicional distinción entre eutanasia activa y pasiva. Es decir, entre la muerte provocada por acción directa del profesional sanitario o por la retirada de los tratamientos que permitirían prolongar la vida. En ambos casos existe un vínculo causal directo que provoca la muerte del paciente y, en ambas situaciones, es el paciente el que toma la decisión siendo perfectamente consciente de que supondrá su fallecimiento.

2) En el suicidio asistido, en cambio, es la propia persona quien termina con su vida, aunque para ello requiera el concurso de otro individuo para hacerse con los medios materiales o intelectuales con los que producir su muerte. Dicha asistencia puede ser proporcionada por personal sanitario o por cualquier persona ajena al ámbito de la salud. Pero, cuando es un facultativo quien ayuda al paciente, hablamos de un suicidio médicamente asistido. Fue, por ejemplo, la opción que tomó Ramón Sampedro, probablemente el rostro más conocido del movimiento por la muerte asistida en España, para poner fin a su propia vida.

3) La limitación del esfuerzo terapéutico (LET) se define como la retirada o la renuncia a iniciar medidas de soporte vital cuando no existe esperanza de recuperación funcional para la persona. O sea, que se descarta prologar la vida biológica a través de mecanismos artificiales (respiradores, etc.) una vez se sabe que el cuerpo del paciente no va a poder mantenerla por sí mismo. En este caso, no se provoca la muerte de la persona, simplemente se permite, dejando que la enfermedad derrote definitivamente al paciente. En estos casos, los matices son realmente difíciles de precisar (el momento en el que no existe posibilidad de recuperación con una mínima calidad de vida, la futilidad o no de conservar los tratamientos de mantenimiento vital...) y requieren de un amplio acuerdo entre todos los profesionales implicados y el paciente o, en caso de que este no esté en condiciones de decidir, los familiares de este. El caso más famoso de LET en España es el de la niña gallega Andrea Lago, afectada por el síndrome de Aicardi-Goutières, una enfermedad neurodegenerativa muy infrecuente. En los meses previos a su muerte, el organismo de Andrea se deterioró de forma irreversible, debiendo ser hidratada y alimentada de 
forma artificial. Los padres de la niña solicitaron que no se siguiera prolongando mecánicamente su vida, lo que consiguieron en octubre del 2015 tras una encarnizada batalla legal que les enfrentó al equipo médico que trataba a su hija.

4) El rechazo del tratamiento contempla el derecho de la persona a negarse a recibir cualquier tratamiento médico que considere puede mermar su calidad de vida, inclusive si este la prolonga durante un tiempo. Es un principio reconocido por el modelo del consentimiento informado, que constituye la base teórica y moral que guía la toma de decisiones en la bioética contemporánea. Como tal, respeta la autonomía del paciente para decidir sobre su cuerpo y su salud. La línea que separa el rechazo del tratamiento y la LET no siempre es sencilla de trazar. Las diferencias principales estriban en el estado de evolución de la enfermedad. En la LET la persona siempre está más allá de la posibilidad de recuperación. El rechazo del tratamiento, por otra parte, puede ser reclamado por el paciente, como sujeto autónomo capaz de tomar las decisiones que estime necesarias respecto a su cuerpo y su salud, desde el primer momento en el que su enfermedad se manifiesta. El ejemplo más conocido de rechazo del tratamiento en España es el de Inmaculada Echeverría (SimónLorda y Barrio-Cantalejo, 2008: 445-451). Echeverría padecía una distrofia muscular progresiva, pero de evolución lenta, lo que mantenía su estado estable durante largos periodos de tiempo. Obligada a residir en el Hospital San Rafael de Granada desde 1997, en el año 2006 solicitó, de forma pública en rueda de prensa, ser desconectada del respirador para no seguir prolongando artificialmente su vida. Tras un intenso debate mediático, jurídico y ético, el Consejo Consultivo de Andalucía y la Comisión Autonómica de Ética e Investigación de Andalucía dictaminaron que la petición de Echeverría era legítima por su derecho de rechazo al tratamiento contemplado en la Ley de Autonomía del Paciente 41/2002 y que los profesionales sanitarios que la ayudasen a cumplirlo no incurrían en delito alguno. Inmaculada Echeverría vio cumplido su deseo en marzo del año 2007.

5) La sedación paliativa de enfermos terminales refiere a la administración a pacientes terminales de fármacos que disminuirán su conciencia de forma profunda y previsiblemente irreversible, como medio de paliar un sufrimiento intenso que no puede aliviarse por otros medios. Es común que acelere la muerte del paciente, pero no se trata de un resultado perseguido por esta práctica, siendo un efecto secundario de la misma. Esto ha llevado a que algunas personas la señalen como una forma de eutanasia encubierta, como sucedió en el tristemente célebre caso del Hospital Severo Ochoa de Madrid. Sin embar- 
go, es una práctica muy común en las unidades de cuidados paliativos de todo el mundo, está considerada una buena práctica sanitaria y requiere siempre del consentimiento informado del paciente o sus representantes.

Como puede observarse, son prácticas próximas pero muy diferentes, que requieren consideraciones legales y morales diferenciadas, entre otras razones porque cada una implica un diferente grado de participación de otras personas en el proceso de muerte. Es por ello también que cada una de ellas es objeto de un distinto grado de aceptación social y viceversa: cada una se ve acompañada de un diferente grado de polémica.

\section{JUSTIFICACIONES}

Con Luc Boltanski y Laurent Thévenot (1991) entenderemos la justificación como la operación discursiva por la cual las personas tratan de legitimar sus opiniones y decisiones vinculándolas a un orden de legitimidad moral (ordre de grandeur). Es decir, a convenciones socialmente aceptadas sobre el bien común, lo correcto e incorrecto. ${ }^{4}$ Podemos identificar dos grandes justificaciones en defensa de la muerte asistida:

1) El principio de autonomía, la libertad de cada ciudadano para decidir soberanamente sobre su cuerpo y su vida. Por tanto, también sobre los tratamientos médicos que afecten al desarrollo de los mismos, incluyendo aquellos referidos a su proceso de muerte, siempre y cuando no dañe a otros, aunque sin extender este último criterio a cualquier tipo de daño moral a terceros, lo que acabaría limitando la libertad de decisión individual hasta extremos que la vaciarían de contenido. Para muchos autores, el auténtico fundamento ético y jurídico en el que se sustentaría el derecho a una muerte digna (Álvarez Galvez, 2002; R. Dworkin et al., 1997; Rivera López, 2003: 79-83; Schramm, 2001; Young, 2014). El más conocido defensor de esta postura es el filósofo norteamericano Ronald Dworkin (1994), quien afirma tajantemente que «el rasgo más relevante de esa cultura [la cultura política occidental] es la creencia en la dignidad humana individual: esto es, que las personas tienen el derecho y la responsabilidad moral de enfrentarse, por sí mismas, a las cuestiones fundamentales acerca del significado y valor de sus propias vidas» (Dworkin, 1994:

\footnotetext{
Boltanski y Thévenot identificaron seis grandes modelos de órdenes de legitimación moral, que denominaron ciudades. Sin embargo, las justificaciones que hemos encontrado para legitimar la muerte asistida no encajan bien en ninguno de los modelos que proponen. Así pues, antes de forzar el modelo, hemos preferido retener la definición de justificación planteada por los autores sin hacer referencia a las ciudades.
} 
214). Para Dworkin, al debatir sobre la eutanasia, como sobre el aborto, no oponemos el derecho a la vida con el derecho a la autonomía. Más bien estaríamos ante diferentes puntos de vista acerca de una preocupación universalmente compartida, como es la integridad de la vida humana. Y, dado que estamos ante concepciones particulares, el principio que debería guiar al legislador es no intervenir, pues los principios morales son, según la doctrina liberal a la que se adhiere Dworkin, estrictamente privados y responsabilidad exclusiva de los individuos.

2) El principio de no sufrimiento, por el que se aspira a que la persona no padezca una muerte dolorosa, innecesariamente alargada o que atente contra su autonomía y dignidad. Esto es, casos en los que la muerte es preferible a la continuación de la vida (Boladeras, 2009; Bouëaseau, 20oo; Tooley, 2005). El supuesto de la preservación de la autonomía es crítico, porque ampliaría los casos en los que se podrían justificar las prácticas vinculadas a la muerte digna más allá del dolor físico intenso. De ahí que sus defensores hablen antes de sufrimiento que de dolor: el sufrimiento es un concepto más amplio que incluiría la pérdida de identidad y autonomía, tanto del moribundo como de sus allegados, conectando así con la noción de dignidad (Bayés, 2003; CohenAlmagor, 20o8; Wijsbek, 2012). Los filósofos australianos Peter Singer y Helga Kushe son los dos autores que más énfasis han puesto en esta vía de fundamentación del derecho a una muerte asistida, llegando a proponer una ética de la calidad de vida que sustituya a la tradicional noción de santidad de la vida (Kushe, 1995, 2001; Singer, 1997).

En realidad, como observó Leonard Wayne Summer (2011), la autonomía y el bienestar del paciente no dejan de ser los dos principios que guían todo tratamiento médico. Normalmente, ambos principios coinciden, pero, cuando no lo hacen, la autonomía debe prevalecer excepto en casos en los que esta no pueda ejercerse, siguiendo el paradigma del consentimiento informado, el más aceptado actualmente en el campo de la bioética, y del que igualmente parten los defensores de la muerte asistida (Wayne Summer, 2011). Ambos argumentos también están presentes en el debate social sobre la muerte asistida, tanto en los movimientos que reclaman su legalización de forma organizada como en los discursos que manejan los ciudadanos favorables al reconocimiento de dichos derechos (Cerrillo Vidal, 2018).

Entre los teóricos del derecho, el posible encaje jurídico del derecho a una muerte asistida ha motivado una discusión compleja. La vida se ha considerado históricamente el primero de los bienes del individuo, la sustancia de todos sus demás derechos. La teoría y la práctica jurídicas han tendido, por esta ra- 
zón, a establecer garantías que blinden la protección de la vida, incluso por encima de la voluntad del propio individuo (Moreno Antón, 2004: 66-68; Ruiz Miguel, 1993: 135-138). En síntesis, en el derecho ha prevalecido una concepción paternalista en la que la muerte solo podía considerarse un disvalor. De ahí que muchos juristas consideren harto problemático reconocer un derecho a morir que iría contra el ordenamiento jurídico y la jurisprudencia. Existirían, además, otros inconvenientes añadidos a reconocer un derecho a la muerte, especialmente si se fundamenta en la libre disposición del sujeto sobre su cuerpo y su vida: moviliza obligaciones de terceros, lo que puede hacer que entre en conflicto con los derechos de estos; dificultaría el control objetivo de su ejercicio, más aún, hasta podría conllevar la generalización del derecho a terminar con la propia vida bajo cualquier circunstancia; algunos autores llegan incluso a considerar que la autonomía personal constituye un fundamento demasiado individualista de los derechos (Méndez Baiges, 2002: 59-66, Rey Martínez, 2008: 161-162; Suárez Llanos, 2012: 335-341).

No obstante, tampoco es cierto que el derecho a la vida sea absoluto. A menudo se ve matizado por otros derechos en los que se considera justificable realizar excepciones, como el homicidio en defensa propia o en caso de conflicto bélico, por no mencionar la pena capital en aquellos países en los que todavía es legal (Moreno Antón, 2004: 68-69, Rey Martínez, 2008: 83-89). El derecho a la muerte asistida tendría así cabida como excepción o desarrollo del derecho a la vida, en función de la articulación de este con otros derechos, como el derecho a la dignidad y a la integridad moral de la persona (Moreno Antón, 2004: 69-72, Rey Martínez, 2008: 89-126). Ciertos estados de deterioro del cuerpo conculcarían la dignidad de la persona o impedirían el libre desarrollo de su personalidad, lo que justificaría que quienes se encuentran en esa situación pudiesen precipitar el fin de sus vidas. $\mathrm{O}$, para resumir con Leonor Suárez Llanos (2012: 341-43), sin calidad de vida el derecho a la vida carecería de sentido.

En fin, como podemos comprobar, desde el campo del derecho se optaría más bien por el principio de no sufrimiento, tal y como lo hemos definido más arriba, como fundamento del derecho a la muerte asistida. Sin embargo, esto no está exento de problemas, pues supone definir el límite de sufrimiento a partir del cual se considera justificado poner fin a una vida. La justificación por el sufrimiento presenta así un problema notable de construcción: definir qué es el sufrimiento. Sin duda, cualquiera de las personas que sostienen esta justificación estaría de acuerdo en considerar una muerte agónica y dolorosa como ejemplo de situación en la que se mostrarían favorables a la muerte asis- 
tida. Ahora bien, el concepto de sufrimiento es más amplio que el dolor físico y abarca una cantidad de condiciones muy diferentes, incluyendo una variada gama de estados psicológicos: pérdida de autoestima, depresión, sentimiento de padecer un deterioro de la identidad, insatisfacción con las circunstancias vitales y un amplio etcétera (Bayés, 2003). ¿En dónde se encuentra el límite del sufrimiento que legitime la muerte asistida? ¿Es suficiente el sufrimiento de una persona con tetraplejia para que esté justificado asistirle en su muerte? ¿O este derecho estaría solo contemplado para enfermos terminales sin esperanza de recuperación?

La justificación por el sufrimiento no contempla estos dilemas, entre otras razones porque no es sistemática. No tiene un principio universalmente aplicable, como la libertad individual en la justificación por la autonomía. Depende de la situación y, más concretamente, de la compasión que desate en el otro el padecimiento de la persona enferma o moribunda. Lo que equivale a decir que se fundamenta menos en el sujeto que sufre que en el que se compadece de su sufrimiento. De esta forma, algunas de las personas que justifican la muerte asistida por el sufrimiento consideran que la inmovilidad forzosa de las personas con tetraplejia es motivo suficiente como para preferir la muerte a su triste situación. Sin embargo, esas mismas personas pueden opinar igualmente que no está justificado el suicidio de una persona que no tuviese problemas de salud física, sino que simplemente se sintiese tan deprimido que se viese incapaz de continuar con su vida. La inmovilidad justifica el derecho a una muerte digna, la tristeza, en cambio, no. ¿Dónde está el límite del sufrimiento? ¿Quién lo establece?

Para decirlo con Singer y Kushe, es preciso definir la calidad de vida que haría legalmente tolerable la muerte asistida. Hacerlo desde estándares externos a la persona afectada resulta enormemente problemático, por muy rigurosa, objetiva y completa que fuese su elaboración. De hecho, sectores contrarios a la legalización de la muerte asistida consideran un ejercicio de totalitarismo todo intento de establecer un canon universalmente aplicable de calidad de vida, en lugar de permitir que sea cada ciudadano el que dote de significado particular a este principio (Gormally, 1997). En este sentido, solo el paciente que lo solicita podría afirmar que padece un sufrimiento tal que deteriora su calidad de vida más allá del límite de lo soportable. Lo que, en la práctica, supone reconocer que, en realidad, es la autonomía, la libre determinación del individuo para hacerse cargo de sus propias decisiones, el fundamento último del derecho a una muerte asistida. 
En efecto, la justificación por la autonomía parte de un principio ético5 susceptible de ser aplicado a cualquier contexto: la libertad individual. Ante la duda, debe prevalecer la voluntad soberana del individuo, con tal de que con su decisión no perjudique a otros ni vulnere la ley. El único debate posible se refiere a las condiciones en las que el individuo no ha expresado claramente su opción o no tiene capacidad de decidir (en cuyo caso el poder de escoger pasaría a la familia, es decir, que permanecería en los ciudadanos). La justificación por la autonomía no se enfrenta, por consiguiente, a ninguna contradicción a la hora de legitimar la muerte asistida. Si las condiciones de vida del individuo le hacen reclamar la muerte, sus deseos deben ser respetados, independientemente de las razones que le hagan expresar su demanda: tanto da que sea el miedo al dolor, la sensación de impotencia derivada de no poder valerse por sí mismo o la merma de las capacidades psíquicas más allá de lo que la persona considera deseable.

En este punto cabría preguntarnos si este razonamiento resulta igual de convincente para el común de la ciudadanía. Al fin y al cabo, no siempre respondemos ante los mejores argumentos: los sesgos en la cognición y la percepción son muchos — disponemos de abundante evidencia al respecto- y su acumulación tiende a viciar la deliberación pública (Kahneman, 2012; Ovejero Lucas, 2013). Uno no menor reside en nuestra preferencia por los relatos. Estamos predispuestos a prestar más atención a la información presentada en forma narrativa que a los datos y a los razonamientos puros (Gibbs, 2012: 8488; McAdams, 1993). En efecto, la fuerza retórica de lo narrativo permite conectar con vivencias universales, refuerza la empatía y resulta más eficiente para organizar y dotar de sentido a la experiencia. Cabría esperar entonces que la justificación por el sufrimiento resultase más apropiada para convencer a una mayoría social de la bondad de la muerte asistida. Después de todo, la historia de una persona suplicando poner fin a su vida por el dolor que padece o las tristes condiciones en las que se encuentra o espera encontrarse pronto ¿no supone un reclamo más contundente y llamativo que la mera apelación a un principio filosófico? ¿No moviliza más nuestras emociones?

\footnotetext{
La distinción entre ética y moral la tomamos de Adolfo Sánchez Vázquez (1999: 17-22). La moral es fundamentalmente práctica y contextual, se refiere a la orientación considerada buena o justa de una acción en la situación concreta. La ética es el conjunto de principios normativos por el que el individuo trata de guiar su vida y, por tanto, los criterios por los que valorará como morales o no sus actos. Moral y ética están así relacionadas, pero la moral es fundamentalmente emotiva mientras que la ética es (o debe ser) racional y reflexiva.
} 
Responder a estas preguntas excedería el espacio de este artículo, pero disponemos de modesta evidencia que parece apuntar en sentido contrario. En un estudio realizado con grupos de discusión (Cerrillo Vidal, 2018: 229-238), se mostraba que los relatos de muertes dolorosas o enfermedades largas y penosas no conseguían convencer a los individuos contrarios a la legalización de la muerte asistida. Estos consideraban que estas experiencias constituían circunstancias particulares, únicas como lo es toda muerte. Cada proceso de muerte es distinto, solo el moribundo sabe qué se siente al morir. La empatía con un moribundo es, en realidad, imposible, porque cada proceso de muerte es irrepetible y el resultado irreversible. Y, si cada caso es único, no cabe establecer patrones, ni intervenir externamente. Pertenece, de hecho, al ámbito de lo privado, así que lo apropiado es interferir lo menos posible. $\mathrm{O}$, dicho de otro modo, el relato no solo no conseguía conectar con marcos universales de bien común, que es el objetivo de la justificación como ya se ha comentado. Ocurría justo lo contrario: se singularizaba la experiencia, recluyéndola a la esfera privada, se le negaba su estatus de problema público o estructural.

En cambio, la justificación por la autonomía, incluso cuando adoptaba formas muy sencillas («que cada uno haga lo que le dé la gana con su vida»), resultaba virtualmente irrefutable para las personas contrarias a la regularización de la muerte asistida. Efectivamente, solo existen dos formas de disputar un discurso que defiende la libertad de elección en lo que al propio cuerpo se refiere: o bien negando la legitimidad del interlocutor o bien negando la idea misma de que el individuo tenga derecho a decidir sobre su vida y su salud. En el primer caso, el contrario a la muerte asistida se definiría a sí mismo como éticamente superior, situándose en una posición por la que se rechazaría la validez de los interlocutores, desestimando que estos pudiesen tener parte de razón. En el segundo, más corriente a juzgar por los resultados del estudio citado, se discute que sea posible o deseable que el individuo tenga libertad de elección, la cual se desplaza hacia la comunidad o hacia Dios, que no deja de ser un avatar de la propia comunidad, como sabemos desde Durkheim (2007). Cualquiera de las dos opciones muestra una cara autoritaria que inmediatamente deslegitima el discurso contrario a la muerte asistida ante personas que tengan mínimamente asimilados el ethos y la identidad individualizada o ciudadana, hegemónicos en sociedades democráticas tardomodernas. En algunos grupos, los participantes en principio opuestos a la muerte asistida terminaban concediendo que, de existir una serie de filtros y controles adecuados (exámenes psicológicos, discusión exhaustiva del caso entre especialistas) y en el caso hipotético de una amplia mejora de las condiciones de vida de los en- 
fermos crónicos y terminales, no podrían negar el derecho a acelerar su muerte de aquellos que así lo siguieran solicitando. Lo contrario supone un paternalismo difícil de digerir por una sociedad que tiene la autonomía individual entre sus valores centrales. La autonomía del sujeto para decidir sobre su propia muerte podía así matizarse, pero no negarse por completo. Como se ha comentado anteriormente, el sufrimiento no disfrutaba de la misma eficacia discursiva.

\section{CONCLUSIÓN}

En resumen, tal y como señalaba Ronald Dworkin, la justificación más sólida para la muerte asistida procede del derecho inalienable de los individuos a hacer lo que deseen con su vida y su muerte. O, lo que es lo mismo, de la libertad individual. Es la conclusión a la que han conducido décadas de debate filosófico y jurídico, y también parece hacerlo la escasa evidencia empírica sobre las preferencias ciudadanas en el proceso final de la vida. La autonomía individual no solo constituye un valor nuclear de la cultura política de las sociedades contemporáneas, además se trata de un principio menos dependiente de contextos particulares de decisión.

El sufrimiento, por su parte, necesita al otro: debo convencer a los demás de que mi padecimiento es tan grande que no existe otra solución que acabar con mi vida. Cuál es el grado de sufrimiento al que debo llegar para que se legitime acabar con mi propia vida es algo que probablemente no pueda ser definido de antemano. Depende de la capacidad de cada caso para movilizar la piedad en los otros. Un problema al que no se enfrenta la autonomía individual: deseo acelerar mi proceso de muerte porque estoy en mi derecho de hacerlo, con independencia de que los demás piensen que mis circunstancias justifiquen tan drástica decisión. Por supuesto, esto no significa que la empatía con los que sufren no juegue un papel importante en la defensa de este peculiar derecho. Lo que resulta innegable es que, por humano que sea este sentimiento, constituye una fundamentación a lo sumo necesaria pero no suficiente de la muerte asistida. 


\section{BIBLIOGRAFÍA}

Aguiar, Fernando, Serrano Del Rosal, Rafael y Sesma, Dolores (2009). Eutanasia y suicidio asistido: un debate necesario. Policy Papers del Centro de Estudios Andaluces, 3. Recuperado de: http://www.centrodeestudiosandaluces.es/datos/factoriaideas/poli cypaper_3.pdf [Consultado el 18 de julio de 2019].

Álvarez Galvez, Iñigo (2002). La eutanasia voluntaria autónoma. Madrid: Dykinson.

Ausín, F. José y Peña, Lorenzo (1998). Derecho a la vida y eutanasia: ¿acortar la vida o acortar la muerte? Anuario de Filosofía del Derecho, XV, 13-30.

Bayés, Ramón (2003). La sociedad contemporánea ante el dolor y la muerte. Humanitas: Humanidades Médicas, 1(1), 53-60.

Boladeras, Margarita (2009). El derecho a no sufrir. Argumentos para la legalización de la eutanasia. Barcelona: Los Libros del Lince.

Boltanski, Luc y Thévenot, Laurent (1991). De la Justification. Les Économies de la Grandeur. Paris: Gallimard.

Bouëaseau G., Marie-Charlotte (2000). La muerte como frontera de sentido: fundamentos para la elaboración de una ética de la medicina paliativa. Ars Médica. Revista de Estudios MédicoHumanísticos, 29(1). doi: 10.11565/arsmed.v29i1.351

Cerrillo Vidal, José Antonio (2018). Una aproximación a los discursos de los andaluces ante la calidad en el morir. Tesis doctoral. Madrid: UNED.

Cohen-Almagor, Raphael (2008). The Right to Die with Dignity: An Argument in Ethics and Law. Health, Law and Policy, 2(1), 2-8.

De Miguel Sánchez, Carolina y López Romero, Alejandro (2006). Eutanasia y suicidio asistido: conceptos generales, situación legal en Europa, Oregón y Australia (I). Medicina Paliativa, 13(4), 207215 .

Douglas, Mary (1973). Pureza y peligro. Un análisis de los conceptos de contaminación y tabú. Madrid: Siglo XXI. 
Dowbiggin, Ian (2007). A Concise History of Euthanasia: Life, Death, God, and Medicine. Lanham: Rowman \& Littlefield.

Durkheim, Emile (2007). Las formas elementales de la vida religiosa. Madrid: Akal.

Dworkin, Ronald (1994). El dominio de la vida. Una discusión acerca del aborto, la eutanasia y la libertad individual. Barcelona: Ariel.

Dworkin, Ronald, Nagel, Thoas, Nozick, Robert, Rawls, Jhon y Scanlon, Thomas (1997). The Case for Legalized Euthanasia. The Philosophers Magazine, 1, 26-31.

Gibbs, Graham (2012). El análisis de datos cualitativos en investigación cualitativa. Madrid: Morata.

Gormally, Luke (1997). Euthanasia and Assisted Suicide: Seven Reasons Why They Should Not Be Legalized. Recuperado de: http://www.bioethics.org.uk/article/1/Euthanasia/Euthanasia\%252 Band\%252BAssisted\%252BSuicide\%253A\%252BSeven\%252BReasons \%252BWhy\%252BThey\%252BShould\%252BNot\%252BBe\%252BLegali zed\%252B\%25281997\%2529 [Consultado el 18 de julio de 2019].

Grillo, Laura S. (2008). Rambu Solo's: The Toraja Cult of Death and Embodied Imagination. En Slattery, Dennis Patrick y Slater, Glen (Eds.). Varieties of Mythic Experience: Essays on Religion, Psyche and Culture (101-133). Carpinteria (Ca): Daimon.

Hertz, Robert (1990). La muerte y la mano derecha. Madrid: Alianza.

Kahneman, Daniel (2012). Pensar rápido, pensar despacio. Barcelona: Penguin Random House.

Kellehear, Allan (2009). Dying Old-and Preferably Alone? Agency, Resistance and Dissent at the End of Life. International Journal of Ageing and Later Life, 4(1), 5-21.

Kushe, Helga (1995). La eutanasia. En Singer, Peter (Ed.). Compendio de Ética (405-416). Madrid: Alianza.

Kushe, Helga (Ed.) (2001). Unsanctifying Human Life: Essays on Ethics. Oxford: Wiley-Blackwell. 
Lawton, Julia (1998). Contemporary Hospice Care: The Sequestration of the Unbounded Body and 'Dirty Dying'. Sociology of Health and Illness, 20(2), 121-143.

Lewis, Paul (2000). The Logic of Christian Theology and the "Right to Die". Issues in Integrative Studies, 18, 65-79.

Manning, Michael (1998). Euthanasia and Physician-assisted Suicide: Killing or Caring? New York: Paulist Press.

McAdams, Dan P. (1993). The Stories We Live by: Personal Myths and the Making of the Self. New York: Guilford Press.

Méndez Baiges, Víctor (2002). Sobre morir. Eutanasias, derechos, razones. Madrid: Trotta.

Moreno Antón, María (2004). Elección de la propia muerte y derecho a la vida: hacia el reconocimiento jurídico del derecho a morir. DS: Derecho y Salud, 12(1), 61-84.

Mystakidou, Kyriaki, Parpa, Efi, Tsilika, Eleni, Katsouda, Emanuela y Vlahos, Lambros (2005). The Evolution of Euthanasia and Its Perceptions in Greek Culture and Civilization. Perspectives in Biology and Medicine, 48(1), 95-104.

Ovejero Lucas, Félix (2013). ¿Idiotas o ciudadanos? El 15-M y la Teoría de la Democracia. Barcelona: Montesinos.

Rey Martínez, Fernando (2008). Eutanasia y derechos fundamentales. Madrid: Tribunal Constitucional.

Rivera López, Eduardo (2003). Eutanasia y autonomía. Humanitas, Humanidades Médicas, 1(1), 79-86.

Ruíz Miguel, Alfonso (1993). Autonomía individual y derecho a la propia vida (un análisis filosófico-jurídico). Revista del Centro de Estudios Constitucionales, 14, 135-165.

Salazar, Arturo (2001). Muerte cerebral. Medwave, 1(05), e3446.

Recuperado de:

http://www.medwave.cl/link.cgi/Medwave/PuestaDia/Cursos/344 6 [Consultado el 18 de julio de 2019].

Sánchez Vázquez, Adolfo (1999). Ética. Barcelona: Crítica. 
Scheper-Hughes, Nancy (200o). Demografía sin números. El contexto económico y cultural de la mortalidad infantil en Brasil. En Viola, Andreu (Ed.). Antropología del Desarrollo. Teoría y Estudios Etnográficos en América Latina (267-299). Barcelona: Paidós.

Schramm, Fermín Roland (2001). ¿Por qué la definición de muerte no sirve para legitimar moralmente la eutanasia y el suicidio asistido? Perspectivas Bioéticas en las Américas, 11(6), 43-54.

Seale, Clive (1995). Dying Alone. Sociology of Health and Illness, 17(3), $376-39^{2}$.

Seale, Clive (1996). Living Alone towards the End of Life. Ageing and Society, 16(1), 75-91.

Seale, Clive (1998). Constructing Death. The Sociology of Death and Bereavement. Cambridge: Cambridge University Press.

Seale, Clive (2004). Media Constructions of Dying Alone: A Form of 'Bad Death'. Social Science and Medicine, 58(5), 967-974.

Simón-Lorda, Pablo y Barrio-Cantalejo, Inés María (2008). El caso de Inmaculada Echeverría: implicaciones éticas y jurídicas. Medicina Intensiva, 32(9), 444-451.

Simón-Lorda, Pablo, Barrio-Cantalejo, Inés María, Alarcos Martínez, Francisco J., Barbero Gutiérrez, Javier, Couceiro, Azucena y Hernando Robles, Pablo (2008). Ética y muerte digna: propuesta de consenso sobre un uso correcto de las palabras. Revista de Calidad Asistencial, $23(6), 271-285$.

Singer, Peter (1997). Repensar la vida y la muerte. El derrumbe de nuestra ética tradicional. Barcelona: Paidós.

Suárez Llanos, Leonor (2012). La ley de la muerte: eutanasias, éticas y derechos. Anuario de Filosofía del Derecho, 28, 323-371.

Thomas, Louis-Vincent (1983). Antropología de la muerte. México: Fondo de Cultura Económica.

Tooley, Michael (2005). In Defense of Voluntary Active Euthanasia and Assisted Suicide. En Cohen, Andrew y Wellman, Cristopher Heath (Eds.). Contemporary Debates in Applied Ethics (161-178). Maden: Blackwell Publishing. 
Wayne Summer, Leonard (2011). Assisted Death. A Study in Ethics and Law. Oxford: Oxford University Press.

Wijsbek, Henri (2012). 'To Thine Own Self Be True': On the Loss of Integrity as a Kind of Suffering. Bioethics, 26(1), 1-7.

Young, Robert (2014). Voluntary Euthanasia. Recuperado de: http:/plato.stanford.edu/entries/euthanasia-voluntary/ [Consultado el 18 de julio de 2019]. 